\title{
Acute Abdomen From Umbilical Hernia Rupture to Flood Syndrome: A Case Report and Review of Literature
}

\author{
Gavin Fang Liu ${ }^{\mathrm{a}, \mathrm{d}}$, Aswin Srinivasan ${ }^{\mathrm{a}}$, Sangeeta Mutnuri ${ }^{\mathrm{b}}$, \\ Muralidhar Reddy Yerramadha ${ }^{\mathrm{c}}$, Mahendra Agraharkar ${ }^{\mathrm{b}}$
}

\begin{abstract}
Flood syndrome is caused by spontaneous rupture of an umbilical hernia in a patient with tense, long-standing ascites. It is a rare complication of hepatic cirrhosis and has a high mortality rate. Flood syndrome is so named because a rush of ascitic fluid often follows the spontaneous umbilical hernia rupture. We present a case of a 39-yearold male patient with a history of alcoholic liver cirrhosis and recurrent ascites who underwent multiple abdominal paracentesis prior to developing an umbilical hernia that eventually ruptured, causing flood syndrome. The authors would like to discuss flood syndrome with a focus on management options.
\end{abstract}

Keywords: Flood syndrome; Ascites; Umbilical hernia rupture; Liver cirrhosis; Spontaneous bacterial peritonitis; Paracentesis

\section{Introduction}

Ascites is a common complication of liver cirrhosis. Up to $20 \%$ of patients with ascites eventually develop umbilical hernias [1-3]. This can occur due to a combination of increased intra-abdominal pressure from ascites and poor nutritional status which then lead to weakening of the anterior abdominal wall muscles $[1,3]$ creating a defect. It is very unusual for an umbilical hernia to rupture, and it can potentially be fatal. Complications of hernia rupture include incarceration of bowel, hypotension secondary to large-volume spontaneous paracentesis and evisceration, development of cellulitis, peritonitis, and sepsis [4]. Postoperative morbidity can be as high as $71 \%$, and mortality ranges between $60 \%$ and $80 \%[1,5$,

Manuscript submitted September 13, 2019, accepted October 1, 2019

aDepartment of Internal Medicine, HCA Houston Healthcare Southeast, Pasadena, TX, USA

bepartment of Nephrology, The University of Texas Medical Branch, Galveston, TX, USA

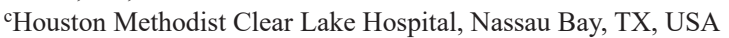

${ }^{d}$ Corresponding Author: Gavin Liu, Department of Internal Medicine, HCA Houston Healthcare Southeast, 4000 Spencer Hwy, Pasadena, TX 77504, USA. Email: fang.liu@rochester.edu

doi: https://doi.org/10.14740/jmc3375
6]. Immediate surgical intervention drops the mortality range to $6-20 \%[1,7,8]$. Flood syndrome refers to the rush of ascitic fluid that follows spontaneous umbilical hernia rupture. Electrolyte abnormalities and other comorbidities must be considered and corrected during management. Prevention and management of these patients have always been controversial. Common surgical treatments are umbilical hernia repair, transjugular intrahepatic portosystemic shunting (TIPS), peritoneovenous shunting (PVS), and concomitant portal venous decompression [1]. Most surgeons are reluctant to undertake surgical treatment of umbilical hernia in patients with cirrhosis and recurrent ascites due to high incidence of postoperative complications including wound dehiscence and evisceration caused by reaccumulation of ascitic fluid, and bleeding due to coagulopathy from liver dysfunction and inadequate synthesis of clotting factors. Wound infection, umbilical hernia recurrence, prolonged ileus, and encephalopathy are just a few more examples of postoperative complications [1]. We present a case of flood syndrome and discuss its prevention and treatment.

\section{Case Report}

A 39-year-old Caucasian gentleman with a past medical history of alcohol abuse, anemia, gastroesophageal reflux disease (GERD), cirrhosis, hematemesis with nausea, and clotting disorder presented to the emergency department with complaints of sudden onset of severe abdominal pain. The patient has a history of decompensated hepatic cirrhosis that started 1 year ago complicated by ascites and a large umbilical hernia. The patient has had multiple paracentesis and had several episodes of spontaneous bacterial peritonitis. His medication list included tramadol and propranolol. Upon admission to the emergency department, he was found to be severely malnourished with significant loss of muscle mass, abdominal distention, and the presence of a large, tender, compressible umbilical hernia. Physical exam showed flank dullness, a fluid wave, and shifting dullness. Vital signs were normal, and he did not show signs of asterixis, hepatic encephalopathy, edema, or fluid overload elsewhere. His white blood cell (WBC) count was high at $10,270 / \mu \mathrm{L}$ and hemoglobin of $10.7 \mathrm{~g} / \mathrm{dL}$, hematocrit of $32.6 \%$, and a platelet count of $130,000 / \mu \mathrm{L}$. Further testing showed hyponatremia at $133 \mathrm{mmol} / \mathrm{L}$ and a total bilirubin of $1.9 \mathrm{mg} / \mathrm{dL}$. Liver function test revealed an albumin of $2.9 \mathrm{~g} /$ 
$\mathrm{dL}$, alkaline phosphatase of $149 \mathrm{IU} / \mathrm{L}$, aspartate aminotransferase (AST) of $64 \mathrm{U} / \mathrm{L}$ and alanine aminotransferase (ALT) of $36 \mathrm{U} / \mathrm{L}$. X-ray confirmed large-volume ascites and the presence of a ventral hernia. Abdominal ultrasound showed cirrhotic liver morphology with large-volume ascites and splenomegaly. Ascitic fluid was yellow, and analysis showed WBC count of $70 / \mu \mathrm{L}$, but was otherwise unremarkable. After being admitted to the hospital, patient was given furosemide $40 \mathrm{mg}$ tablet daily and spironolactone $100 \mathrm{mg}$ tablet daily. The patient was admitted to the hospital for further management. Surgical consultation was sought, and since the patient was a very high-risk surgical candidate with loss of muscle mass, surgical intervention was not planned. The patient chose supportive care and prophylactic antibiotics based on infectious disease recommendations. An ostomy pouch was placed over the hernia to prevent bacterial contamination and peritonitis as well as to collect the ascitic fluid. The patient's severe abdominal pain subsided and was discharged to follow up with his primary care physician.

Three months after the patient was discharged, he came back to the hospital with worsening symptoms because he could not afford the ostomy pouches. He was not compliant with his follow-ups. His umbilical hernia appeared to have worsened, and ascitic fluid was leaking out from multiple areas on his hernia, and there were also multiple areas of skin necrosis. Since the patient was deemed a high-risk surgical candidate, he was, again, not cleared for surgery. The patient opted for conservative care and prophylactic antibiotics according to infectious disease suggestions. After symptoms improved, he was instructed on being more compliant and then subsequently discharged.

\section{Discussion}

Our patient has had several hospital admissions due to refractory ascites, which was complicated by spontaneous bacterial peritonitis, hyponatremia, and renal failure. Surgical intervention was declined by several surgeons due to a high risk of complications such as postoperative wound dehiscence, bleeding, wound infection, prolonged ileus, and encephalopathy. Umbilical hernia repair is also contraindicated in patients with patent umbilical vein as it could lead to ligation of umbilical vein resulting in obstructed portal circulation outflow which may cause acute portal vein thrombosis and subsequent acute liver failure [9-11]. TIPS is the most widely used surgical method. However, TIPS has a wide variety of complications and risks such as hemorrhage, encephalopathy, TIPS dysfunction, and liver failure. Its morbidity risk can be as high as $20 \%$ [12], and it is not widely available as it is only performed in specialized centers. This suggests that conservative management as the preferred treatment. However, conservative measures are also associated with a high risk of spontaneous bacterial peritonitis.

Our patient has had multiple episodes of spontaneous bacterial peritonitis in the past. After the occurrence of flood syndrome, the need for paracentesis decreases or ceases due to a spontaneous drainage of ascitic fluid. However, due to exposure of the ascitic fluid to the outside environment, the risk of peritonitis is increased. Placement of an ostomy pouch may significantly contain the danger of peritonitis but does not completely negate the risk. Therefore, the prevention of flood syndrome is of utmost importance. The preventive measures are to decrease ascitic fluid formation using medical therapies like diuretics including furosemide and spironolactone and to perform frequent and regular paracentesis to avoid the development of umbilical hernia. Other methods of prevention include repeated patient education regarding the complications of cirrhosis, ascites, dietary salt and fluid restriction in addition to avoidance of alcohol and nonsteroidal anti-inflammatory drugs (NSAIDs). A hernia truss is controversial and may make the hernia worse and should only be used temporarily if at all. It is also uncomfortable, and therefore, patient compliance is poor [13]. Once ascites develops and is not adequately controlled by medications (like diuretics), frequent and regular paracentesis remains the preferred preventive measure.

\section{Conclusions}

Flood syndrome is a rare and potentially fatal complication of ascites and liver cirrhosis. It may lead to complications such as peritonitis and evisceration. Unfortunately, there are no good measures to treat flood syndrome, therefore it must be prevented from occurring. Effective preventative methods include salt and fluid restriction, diuretics, frequent and regular therapeutic paracentesis to prevent filling of ascitic fluid.

\section{Acknowledgments}

None to declare.

\section{Financial Disclosure}

The authors have no financial disclosure to declare.

\section{Conflict of Interest}

None to declare.

\section{Informed Consent}

Informed consent was obtained.

\section{Author Contributions}

Mahendra Agraharkar contributed to conception and design of the study. Gavin Liu performed the study. Gavin Liu, Mahendra Agraharkar, Sangeeta Mutnuri, and Aswin Srinivasan con- 
tributed to drafting and revising the article. Muralidhar Reddy Yerramadha contributed to acquisition of data.

\section{References}

1. Chatzizacharias NA, Bradley JA, Harper S, Butler A, Jah A, Huguet E, Praseedom RK, et al. Successful surgical management of ruptured umbilical hernias in cirrhotic patients. World J Gastroenterol. 2015;21(10):31093113.

2. Dokmak S, Aussilhou B, Belghiti J. Umbilical hernias and cirrhose. J Visc Surg. 2012;149(5 Suppl):e32-39.

3. Belghiti J, Durand F. Abdominal wall hernias in the setting of cirrhosis. Semin Liver Dis. 1997;17(3):219-226.

4. DeLuca IJ, Grossman ME. Flood syndrome. JAAD Case Rep. 2015;1(1):5-6.

5. Baron HC. Umbilical hernia secondary to cirrhosis of the liver. Complications of surgical correction. N Engl J Med. 1960;263:824-828.

6. Lemmer JH, Strodel WE, Knol JA, Eckhauser FE. Management of spontaneous umbilical hernia disruption in the cirrhotic patient. Ann Surg. 1983;198(1):30-34.
7. Kirkpatrick S, Schubert T. Umbilical hernia rupture in cirrhotics with ascites. Dig Dis Sci. 1988;33(6):762-765.

8. Telem DA, Schiano T, Divino CM. Complicated hernia presentation in patients with advanced cirrhosis and refractory ascites: management and outcome. Surgery. 2010;148(3):538-543.

9. Oosterwijk PR, Kouw E, de Vos tot Nederveen Cappel WH. Two cases of spontaneous rupture of an umbilical hernia, a rare complication of portal hypertension. Arch Clin Gastroenterol. 2017;3(3):071-073.

10. Marsman HA, Heisterkamp J, Halm JA, Tilanus HW, Metselaar HJ, Kazemier G. Management in patients with liver cirrhosis and an umbilical hernia. Surgery. 2007;142(3):372-375.

11. Triantos CK, Kehagias I, Nikolopoulou V, Burroughs AK. Surgical repair of umbilical hernias in cirrhosis with ascites. Am J Med Sci. 2011;341(3):222-226.

12. Suhocki PV, Lungren MP, Kapoor B, Kim CY. Transjugular intrahepatic portosystemic shunt complications: prevention and management. Semin Intervent Radiol. 2015;32(2):123-132.

13. Kingsnorth A, LeBlanc K. Hernias: inguinal and incisional. Lancet. 2003;362(9395):1561-1571. 